\title{
Computational modeling of chromosome re-replication in mutant strains of fission yeast
}

\author{
Béla Novák ${ }^{a, *}$ and John J. Tyson ${ }^{b}$ \\ aDepartment of Biochemistry, University of Oxford, Oxford OX1 3QU, UK; b Department of Biological Sciences, \\ Virginia Tech, Blacksburg, VA 24061, USA
}

\begin{abstract}
Typically cells replicate their genome only once per division cycle, but under some circumstances, both natural and unnatural, cells synthesize an overabundance of DNA, either in a disorganized manner ("overreplication") or by a systematic doubling of chromosome number ("endoreplication"). These variations on the theme of DNA replication and division have been studied in strains of fission yeast, Schizosaccharomyces pombe, carrying mutations that interfere with the function of mitotic cyclin-dependent kinase (Cdk1:Cdc13) without impeding the roles of DNA-replication loading factor (Cdc18) and S-phase cyclin-dependent kinase (Cdk1:Cig2). Some of these mutations support endoreplication, and some overreplication. In this paper, we propose a dynamical model of the interactions among the proteins governing DNA replication and cell division in fission yeast. By computational simulations of the mathematical model, we account for the observed phenotypes of these re-replicating mutants, and by theoretical analysis of the dynamical system, we provide insight into the molecular distinctions between overreplicating and endoreplicating cells. In the case of induced overproduction of regulatory proteins, our model predicts that cells first switch from normal mitotic cell cycles to growth-controlled endoreplication, and ultimately to disorganized overreplication, parallel to the slow increase of protein to very high levels.
\end{abstract}

\begin{abstract}
Monitoring Editor
Alex Mogilner

New York University
\end{abstract}

Received: Sep 24, 2020

Revised: Jan 25, 2021

Accepted: Jan 25, 2021

\section{INTRODUCTION}

The eukaryotic cell cycle is characterized by strict alternation of DNA replication during $\mathrm{S}$ phase and chromosome segregation in mitosis (M phase). During mitotic cell divisions, each $\mathrm{S}$ phase is followed by mitosis and every $M$ phase is preceded by the replication of all chromosomes. Both of these chromosomal events are triggered by periodic activation of cyclin-dependent kinases (Cdk) in complex with their regulatory subunits, cyclins. Most eukaryotic cells use different Cdk:cyclin pairs to trigger DNA replication and entry into mitosis (Morgan, 2007). The lower eukaryote, Schizosaccharomyces pombe (fission yeast) uses the same Cdk (called Cdk1) in both

\footnotetext{
This article was published online ahead of print in MBoC in Press (http://www .molbiolcell.org/cgi/doi/10.1091/mbc.E20-09-0610) on February 3, 2021. *Address correspondence to: Béla Novák (bela.novak@bioch.ox.ac.uk). Abbreviations used: Cdk, cyclin-dependent kinase; DPR, DNA:protein ratio; MBF, Mlul box-binding factor; MCM, minichromosome maintenance; NFL, negative feedback loop; SCF, Skp-Cullin-Fbox; SK, starter kinase; S-R, Signal-Response; $\mathrm{TFI}$, transcription factor inhibitor.

(C) 2021 Novák and Tyson. This article is distributed by The American Society for Cell Biology under license from the author(s). Two months after publication it is available to the public under an Attribution-Noncommercial-Share Alike 3.0 Unported Creative Commons License (http://creativecommons.org/licenses/by-nc-sa/3.0).

"ASCB®," "The American Society for Cell Biology®," and "Molecular Biology of

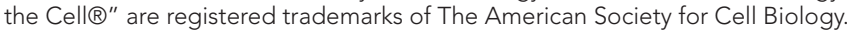

complexes; Cdk1 associates with S-phase cyclin (Cig2) to initiate DNA replication, while its complex with mitotic cyclin (Cdc13) brings about M phase (Nurse, 1990; Mondesert et al., 1996). The activities of Cdk1:Cig2 and Cdk1:Cdc13 oscillate during the cell cycle, with peaks during DNA replication and mitosis, respectively (Figure 1A). The alternating oscillations of Cdk1:Cig2 and Cdk1:Cdc13 generate a steadily increasing activity of Cdk1 (the sum of Cig2- and Cdc13dependent kinase activities) from the beginning of DNA replication until the end of M phase (Moreno et al., 1989; Fisher and Nurse, 1996; Stern and Nurse, 1996). After triggering DNA synthesis from multiple origins of replication, the rising activity of Cdk1 blocks origins from firing a second time until the cell exits from mitosis (Diffley, 2004). At the end of mitosis, Cdk1:Cdc13 activity is reduced to a value close to zero by proteasomal degradation of $\mathrm{Cdc} 13$, which is triggered by abrupt polyubiquitinylation of $\mathrm{Cdc} 13$ by an E3 ligase, the Anaphase Promoting Complex/Cyclosome (APC/C). In the absence of any Cdk1 activity during the following G1 phase, replication origins can be relicensed for another round of DNA synthesis by the action of the Cdc18 "loading-factor" (Kelly et al., 1993). Subsequently, a new batch of Cig2 is synthesized, and Cdk1:Cig2 activity initiates another $\mathrm{S}$ phase by activation of licensed replication origins. By temporally separating the licensing and activation of replication origins, the oscillation of overall Cdk1 activity between very low and 
A

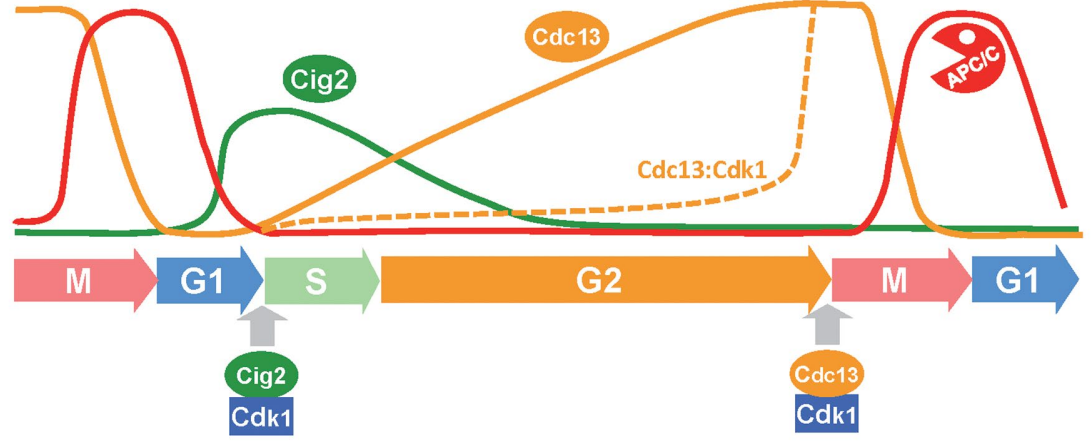

B

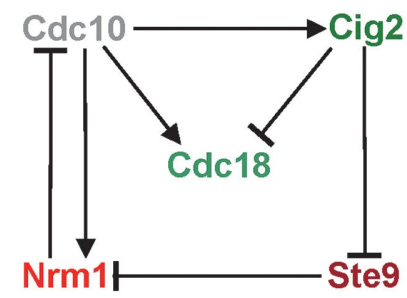

C

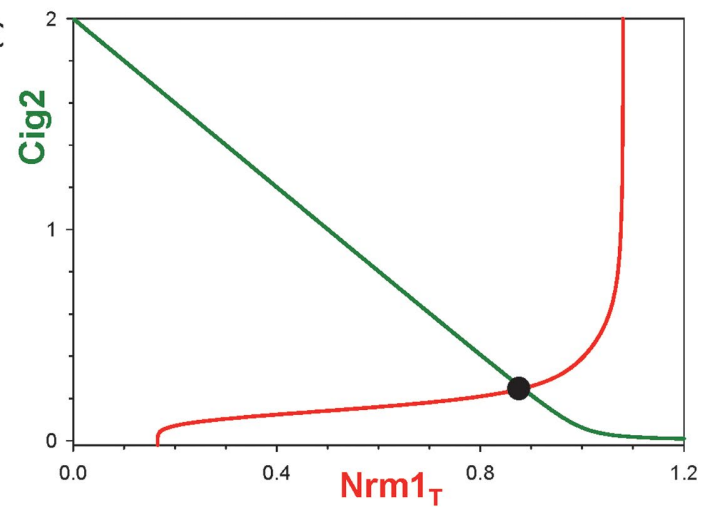

FIGURE 1: The core transcriptional negative feedback of fission yeast. (A) Fluctuations of cell cycle regulators during wild-type fission yeast cell cycle (schematic). (B) Influence diagram for Cdc10 downstream targets. (C) The negative feedback on the Nrm1 ${ }_{\mathrm{T}}$-Cig2 plane. Each dynamic variable is in steady state along its balance curve. Since Nrm1 inhibits the transcription of Cig2, Cig2 level is decreasing with $\mathrm{Nrm}_{\mathrm{T}}$ levels. Cig2 inhibits $\mathrm{Nrm} 1$ degradation, therefore $\mathrm{Nrm} 1_{\mathrm{T}}$ level shows a switchlike increase with Cig2. At the intersection of the two balance curves, the whole network is in steady state.

high values guarantees a single genome duplication between two successive chromosome segregations. In this manner, the strict alternation between $\mathrm{S}$ and $\mathrm{M}$ phases of the mitotic cycle in wild-type fission yeast is strongly coupled to the alternating peaks of Cdk1:Cig2 and Cdk1:Cdc13 oscillations.

If an essential chromosomal event (e.g., DNA replication or mitotic spindle formation) becomes compromised, then further progression through the cell cycle must be suspended until the problem can be resolved. These potential problems are monitored by surveillance mechanisms that, when triggered, block the activation of the kinase complex (Cdk1:Cig2 or Cdk1:Cdc13) responsible to initiate the next event in the chromosome replication/division cycle. In addition, the oscillations of both Cdk1:cyclin complexes are controlled by a "cell size" checkpoint. The critical size threshold permissive for oscillation is higher for the M-phase kinase than for the S-phase kinase (Wood and Nurse, 2015).

Interestingly, the normally interlinked oscillations of Cig2- and Cdc13-dependent kinases can run independently from each other, but these independent Cdk1 oscillations have different physiological outcomes. Because Cdc13 is the only essential cyclin for the fission yeast cell cycle, it can, in the absence of S-phase cyclin (i.e., in cig2 $\Delta$ cells), drive both DNA replication and mitosis (Fisher and Nurse, 1996; Martin-Castellanos et al., 2000; Coudreuse and Nurse, 2010). Since the activity of Cdk1 required for $S$ phase is lower than for $M$ phase (Coudreuse and Nurse, 2010), the oscillating activity of Cdk1:Cdc13 by itself guarantees that initiation of DNA replication always precedes mitosis. Because the rate of synthesis of $\mathrm{Cdc} 13$ is uniform throughout the cell cycle, it is an underlying proteolytic

negative feedback loop (NFL; through APC/C) that is responsible for the oscillation of $\mathrm{Cdc} 13$ level. The amplitude of the oscillation of Cdk1:Cdc13 activity is boosted by a posttranslational positive feedback loop whose activation is linked to a critical cell size (Tyson et al., 2002; Gerard et al., 2015).

Because $c d c 13$ is an essential gene, the consequences of eliminating $\mathrm{Cdc} 13$ protein are studied in fission yeast cells carrying the cdc $13^{+}$gene driven by a thiamine-repressible promoter. These cells, growing in the presence of thiamine, cannot enter mitosis, but their Cdk1:Cig2 activity continues to oscillate (Wuarin et al., 2002). Since these persisting Cdk1:Cig2 oscillations do not perturb the temporal separation of replication-origin licensing and activation, Cdc13depleted cells execute periodic, discrete rounds of DNA replication without mitosis and cell division (Hayles et al., 1994). During these "endoreplication" cycles, the activation of Cig2-dependent kinase ("Cig2kinase", hereafter) maintains its correlation to cell size, as indicated by a characteristic DNA:protein ratio (DPR) at initiation of periodic DNA replications (Kiang et al., 2009).

DNA replication is also uncoupled from mitosis in cdc18 op cells that overexpress Cdc18 protein, a loading factor for DNA replication (Nishitani and Nurse, 1995). Cdc18 is also an inhibitor of Cdc13-kinase (Greenwood et al., 1998; Hermand and Nurse, 2007), which compromises the system of replication-origin licensing and activation. Consequently, in cells overexpressing Cdc18, DNA origins of replication are constitutively licensed and activated (Kiang et al., 2010), but they have insufficient Cdk1 activity to enter mitosis. These cells "overreplicate" their genome in a temporally disorganized manner. We also discuss situations when Cdc13-kinase activity is driven to low level by overexpression of Ste9, a component of the Cdc13 degradation pathway (Yamaguchi et al., 1997; Kitamura et al., 1998), or by overexpression of Rum1, a strong stoichiometric inhibitor of Cdk1:Cdc13 (Moreno and Nurse, 1994).

In this paper, we present a dynamical model of the Cdk1:Cig2 oscillations that regulate fission-yeast DNA replication, with special emphasis on endoreplication cycles. We build our model in a modular manner, starting with the transcriptional NFL that is at the heart of the oscillatory mechanism. Nonlinear negative feedback is necessary, but not sufficient, for limit cycle oscillations, because the feedback signal must be "delayed" in time. As described in Novak and Tyson (2008), the "delay" could be an explicit time delay caused, for example, by a long feedback loop, or it could be a functional delay caused, for example, by positive feedback loop(s) that amplify the action of the activator or the inhibitor in the NFL. Following this line of thinking, we propose two potential positive feedback mechanisms-not mutually exclusive-that could provide the necessary time delay to make the NFL oscillate. Next, we propose mechanisms for the entrainment of Cig2 oscillations by the DPR during endoreplication cycles and by Cdk1:Cdc13 oscillations during mitotic cycles. Finally, we propose a simple model for cycling of replication origins (loaded $\rightarrow$ fired $\rightarrow$ replicated $\rightarrow$ unloaded $\rightarrow \ldots$ ) and 
use it to account for the difference between aperiodic DNA overreplication and periodic endoreplication.

\section{RESULTS}

\section{The core NFL}

Eukaryotic DNA replication origins must be "licensed" by MCM (minichromosome maintenance) proteins before they become activated by Cdk1:cyclin complexes at initiation. In fission yeast, the role of DNA-replication loading factor is played by Cdc18 (Kelly et al., 1993) and Cdt1 (Nishitani et al., 2000). The transcription of both loading factors and of S-phase cyclin, Cig2, is induced by a transcription factor called MBF (Mlul cell cycle box-binding factor), which contains the Cdc10 protein as a core component (Reymond et al., 1993). The activity of MBF fluctuates during both mitotic (Ayte et al., 2001; Rustici et al., 2004) and endoreplication cycles (Kiang et al., 2009), being activated before and inactivated after DNA replication. The oscillation of MBF activity is caused by a transcriptional NFL created by several protein products of MBFdependent transcription: Cig2, Nrm1 and Yox1 (Ayte et al., 2001; Bertoli et al., 2013). The Nrm1:Yox1 complex is thought to be a stoichiometric inhibitor of MBF (Cdc10) after S phase (Bertoli et al., 2013), creating a two-component NFL (Cdc10 $\rightarrow$ Nrm1 $\dashv$ Cdc10; Figure 1B). In addition, Nrm1 protein is targeted for degradation by APC/C:Ste9 (Bertoli et al., 2013), and Cdk1:Cig2 kinase inactivates APC/C:Ste9 by multisite phosphorylation of Ste9 (Blanco et al., 2000; Yamaguchi et al., 2000), creating a longer NFL $(\mathrm{Cdc10} \rightarrow \mathrm{Cig} 2 \dashv$ Ste9 $\dashv \mathrm{Nrm} 1 \dashv \mathrm{Cdc10}$; Figure 1B). During endorepli-

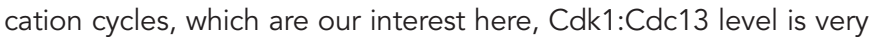
low (cdc $13^{+}$transcription is repressed by thiamine); therefore, the phosphorylation-dependent inactivation of Ste9 relies exclusively on Cdk1:Cig2. The S-phase Cdk1 complex can inactivate Ste9 efficiently because Cig2 is not targeted for degradation by APC/C:Ste9 (Blanco et al., 2000). Cdk1:Cig2 also phosphorylates Cdc18, which targets the loading factor for rapid ubiquitinylation-dependent degradation (Jallepalli et al., 1997). (In the following, we focus on Cdc18 regulation, neglecting $\mathrm{Cdt1}$.) Notice that, in our model in Figure 1B, Cig2-kinase indirectly inhibits Cdc10 by inhibiting degradation of Nrm1 through inactivation of Ste9. In summary, the level of Nrm1 is up-regulated by MBF-dependent transcription through a coherent feedforward loop that increases $\mathrm{Nrm} 1$ synthesis (Cdc10 $\rightarrow \mathrm{Nrm} 1$ ) and inhibits its degradation (Cdc10 $\rightarrow$ Cig2 $\dashv$ Ste9 $\dashv \mathrm{Nrm} 1)$.

The inactivation of Ste9 by Cdk1:Cig2-dependent multisite phosphorylation and the stoichiometric inhibition of $\mathrm{Cdc} 10$ by Nrm1:Yox1 introduce strong nonlinearities into the transcriptional NFL. These nonlinear interactions can be conveniently illustrated by balance curves on an [Nrm1 $]_{T}-[\mathrm{Cig} 2]$ coordinate system, which is called a "phase plane" (Figure 1C) (Novak and Tyson, 2008). A balance curve indicates the effect of one variable on the steadystate level of the other. The balance curve of Cig2 (the green curve) decreases with increasing Nrm1, because Nrm1 inhibits Cdc10dependent transcription of Cig2. In contrast, the steady-state level of Nrm1 shows a switchlike increase above a threshold level of Cig2, the level at which Cig2-kinase effectively inactivates APC/C:Ste9.

A NFL with sufficient nonlinearities and time delay can exhibit sustained oscillation over a particular range of kinetic parameters (Novak and Tyson, 2008). The longer NFL in Figure 1B has four components, but its oscillatory potential is suppressed because the phosphorylation of Ste9 by Cig2-kinase and the inhibition of Cdc10 by Nrm1-binding are fast reactions compared with synthesis and degradation of Cig2 and Nrm1 proteins. By making pseudo-steadystate assumptions on Ste9 and Cdc10, we reduce the fourdimensional dynamical system to two dimensions (the Cig2-Nrm1 phase plane in Figure 1C). The steady state of the reduced system is found at the intersection of the two balance curves, where both $\mathrm{Cig} 2$ and $\mathrm{Nrm} 1$ are unchanging in time. This steady state is globally stable. According to numerical simulations (not shown), all components approach their steady state values monotonically, except Cdc18, which shows a transient peak because Cdc18 level is regulated by an incoherent feedforward loop: Cdc10 directly activates synthesis of $\mathrm{Cdc} 18$ and indirectly promotes its degradation (through Cig2-kinase). We note here that proteasomal degradation of Cdc18 and $\mathrm{Cig} 2$ is initiated by polyubiquitinylation of the phosphorylated substrates (Cdc18-P, Cig2-P) by the Skp-Cullin-Fbox (SCF) ubiquitin ligase (Kominami and Toda, 1997).

Because the time delays in our transcriptional NFLs (Cdc10 $\rightarrow \mathrm{Nrm} 1 \dashv \mathrm{Cdc} 10$ and $\mathrm{Cdc} 10 \rightarrow \mathrm{Cig} 2 \dashv \mathrm{Ste} 9 \dashv \mathrm{Nrm} 1 \dashv \mathrm{Cdc} 10)$ are not long enough to induce oscillations, we consider positive feedback loops as alternatives to destabilize the steady state and generate relaxation-type oscillatory behavior in the model. In the next two subsections, we explore two positive feedback loops in the Cig2-kinase control network.

\section{Positive feedback regulation of Cig2 activity}

Cdk1:cyclin complexes are regulated by transient, inhibitory phosphorylations of their Cdk1 subunit that supress their protein kinase activities. In fission yeast, the inhibitory Cdk1-phosphorylations provide the dominant control over mitotic Cdk1:Cdc13 complexes (Nurse, 1990). Moreover, the S-phase inducing Cdk1:Cig2 complex also undergoes transient, inhibitory phosphorylations, which persist if DNA replication is inhibited (Zarzov et al., 2002; Hermand and Nurse, 2007). In fission yeast, two inhibitory kinases, Wee1 (Russell and Nurse, 1987) and Mik1 (Lundgren et al., 1991), and two activatory protein phosphatases (Cdc25 and Pyp3) are responsible for the reversible, inhibitory phosphorylations of Cdk1:cyclin complexes (Russell and Nurse, 1986; Millar et al., 1992). The phenotypes of loss-of-function mutants suggest that Wee1 and Mik1 are acting, respectively, in late $\mathrm{G} 2$ phase and in S phase of the cell cycle (Nurse, 1975; Lundgren et al., 1991). Based on these observation, we propose that Mik1 is the dominant inhibitory kinase for the Cdk1:Cig2 complex (Ng et al., 2001). For simplicity, we assume constant activity for the activatory phosphatase (Cdc25 and/or Pyp3). The level of Mik1 protein fluctuates during the cell cycle, showing a peak during S phase (Christensen et al., 2000), which we explain by SCF-induced degradation of Mik1 after it is phosphorylated by Cig2-kinase, similar to the case of Cdc18 degradation. Briefly, in our model, Cdk1:Cig2 and Mik1 mutually inhibit each other, which creates a positive feedback loop (Figure 2A).

The positive feedback loop puts a "kink" in the Nrm1-balance curve (the red curve in Figure 2B) and thereby creates the possibility of oscillations in the Cig2-Nrm1 NFL (Novak and Tyson, 2008). The Nrm1-balance curve is N-shaped because more Cig2 is required to turn off Ste9 and up-regulate Nrm1 than is needed to maintain $\mathrm{Nrm} 1$ at high levels. Notice that we plot on the $y$-axis the total Cdk1:Cig2 level, [Cig2]T, which is the sum of active and inactive (Cdk1-phosphorylated) complexes. This antagonistic relationship between Mik1 and Cdk1:Cig2 generates an effective time delay to make the transcriptional NFL oscillatory (Figure 2C). During the oscillation, Mik1 inactivates Cdk1:Cig2 first, and only later is Cdk1:Cig2 able to promote Mik1 degradation. The delayed activation of Cdk1:Cig2 by inhibitory phosphorylation could be very significant for the control of DNA replication by providing a time window for $\mathrm{Cdc} 18$ to licence replication origins before Cdk1:Cig2 activity appears (Figure 2C). Our model predicts that inhibitory phosphorylation 
A
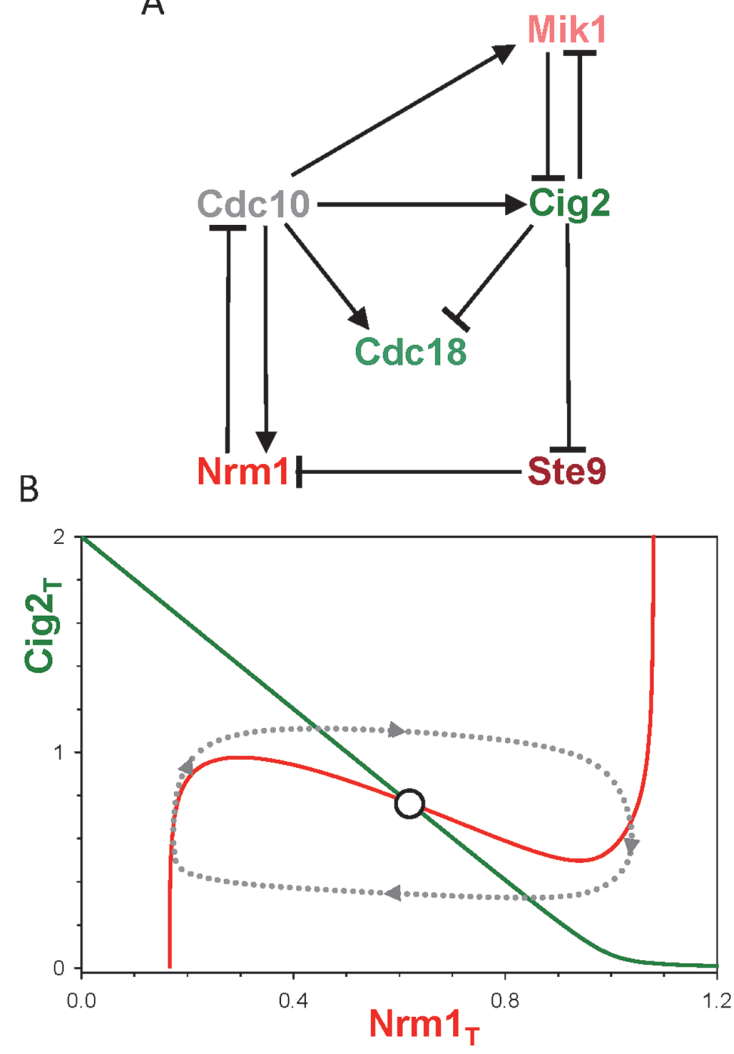

C

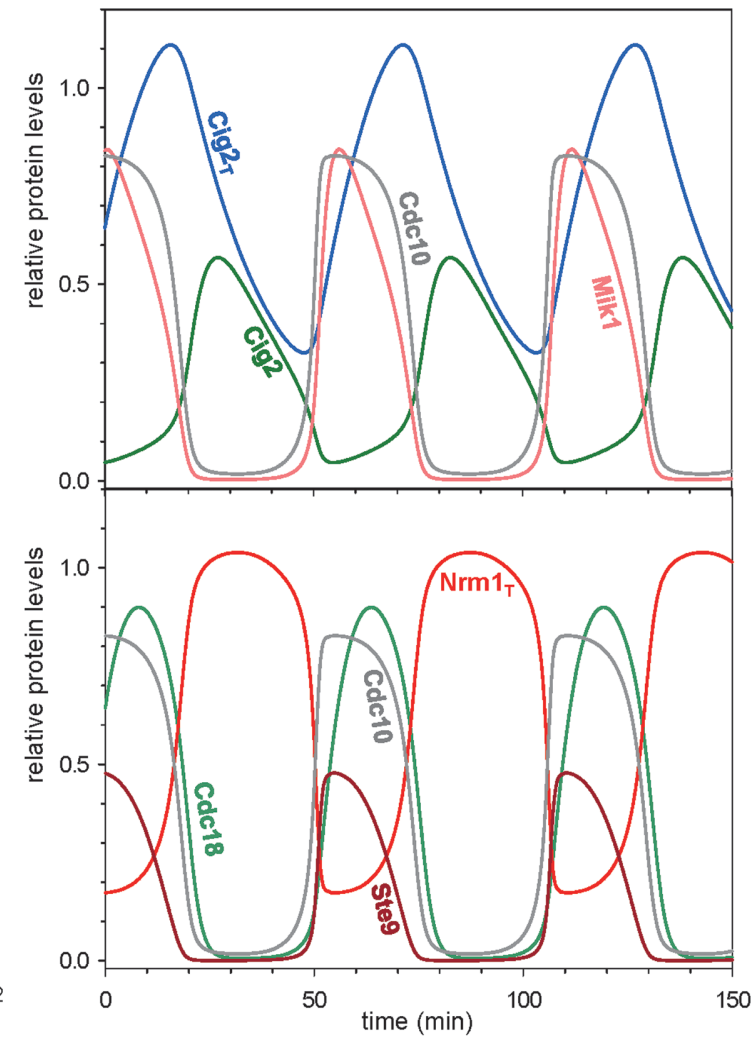

FIGURE 2: Positive feedback regulation of Cig2-kinase. (A) Influence diagram. (B) Phase plane portrait: the positive feedback makes $\mathrm{Nrm}_{\mathrm{T}}$ a bistable switch indicated by its $\mathrm{N}$-shaped balance curve (red). The limit cycle oscillation is indicated by the dotted closed orbit. (C) Numerical simulation of the network shown in A.

of Cdk1:Cig2 by Mik1-kinase could be an essential step in creating Cig2 oscillations and genome reduplication.

\section{Positive feedback regulation of Cdc10 transcription factor}

In the previous example, the transcriptional NFL was made oscillatory by positive feedback regulation of Cig2 activity. The other possibility for introducing positive feedback is autoactivation of the Cdc10 transcription factor mediated by a "transcription factor inhibitor" (TFI) (Supplemental Figure S1A). The paradigm for this hypothetical TFI is the regulation of transcription factors E2F and SBF in mammalian cells and budding yeast, respectively (see, e.g., Bertoli et al., 2013). Both E2F and SBF are inhibited by stoichiometric binding partners (retinoblastoma protein and Whi5, respectively), which become inactivated by Cdk:cyclin complexes (Cdk2:CycE and $\mathrm{Cdk} 1: \mathrm{Cln} 2$, respectively) that are up-regulated by their respective transcription factors. Since fission yeast also has a Whi5 gene and protein, it is reasonable to hypothesize a stoichiometric inhibitor (TFI) of Cdc10 that controls the activity of MBF. We assume that unphosphorylated TFI binds reversibly and inhibits MBF (Cdc10) and the inhibition is terminated by phosphorylation of TFI, which is carried out by a protein kinase up-regulated by Cdc10. Since the Cdc10 target responsible for inactivation of TFI is unknown, we assume for the time being that Cig2-kinase is responsible for this reaction.

In this scenario, MBF has two stoichiometric inhibitors, which are regulated differently: activation of $\mathrm{Cdc10}$ inhibits TFI while it activates Nrm1 directly (synthesis) and indirectly through Cig2 and Ste9. The positive feedback loop between Cdc10 and TFI puts a kink in the Cig2 balance curve (the green curve in Supplemental Figure S1B).
On the upper and the lower branches of the Cig2 balance curve TFI is phosphorylated (inactive) and dephosphorylated (active), respectively. The bistable region is bounded by two different Nrm1 thresholds. The Nrm1 threshold required to induce dephosphorylation of TFI is higher than the threshold required to maintain TFI in the active, dephosphorylated state. If the red balance curve intersects the Z-shaped green balance curve on the middle branch of the " $Z$," then the system exhibits limit cycle oscillations with periodic phosphorylation and dephosphorylation of TFI (Supplemental Figure $\mathrm{S} 1 \mathrm{C})$. The oscillations of Cig2, Nrm1, and Cdc10 are quite acceptable in this model, but the waveform of Cdc18 oscillations is unacceptable. Because there is little time delay between the activation of Cdc10 and of Cig2, Cdc18 does not rise to a high, sustained level to effectively load MCMs on replication origins.

The two positive feedback mechanisms controlling Cdc10 and Cig2 activities are not mutually exclusive; most likely, they operate in parallel in the same regulatory network (Figure 3A). With both positive feedbacks in place, both Nrm1 and Cig2 balance curves are kinked ( $\mathrm{N}$ - and Z-shaped, respectively, in Figure 3B), and the oscillations preserve the good dynamic features of both scenarios (Figure 3C). Although the positive feedback on Cdc10 seems to be inconsequential in the oscillation, it plays an important physiological role, as we discuss in a subsequent session.

\section{Control of replication origin dynamics}

Cdk1:cyclin oscillations could drive a complete round of eukaryotic genome replication if multiple replication origins fire once and only once, which requires that Cdk1 activity both activates and inhibits DNA replication (Diffley, 2004, 2011). To test how Cig2-kinase 

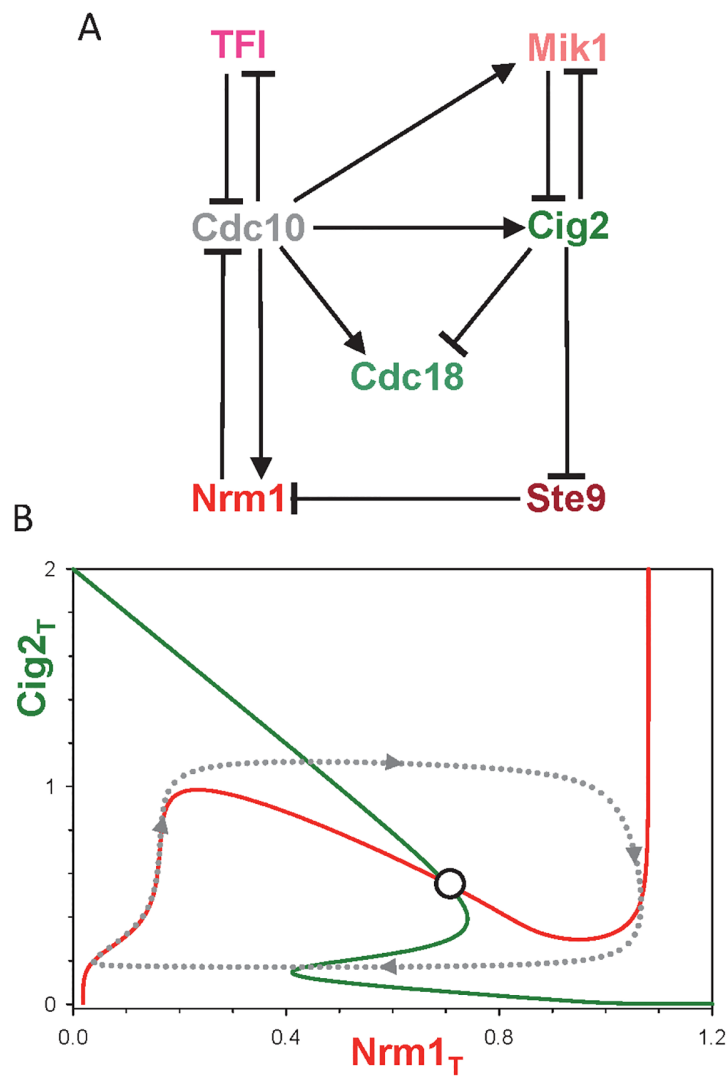

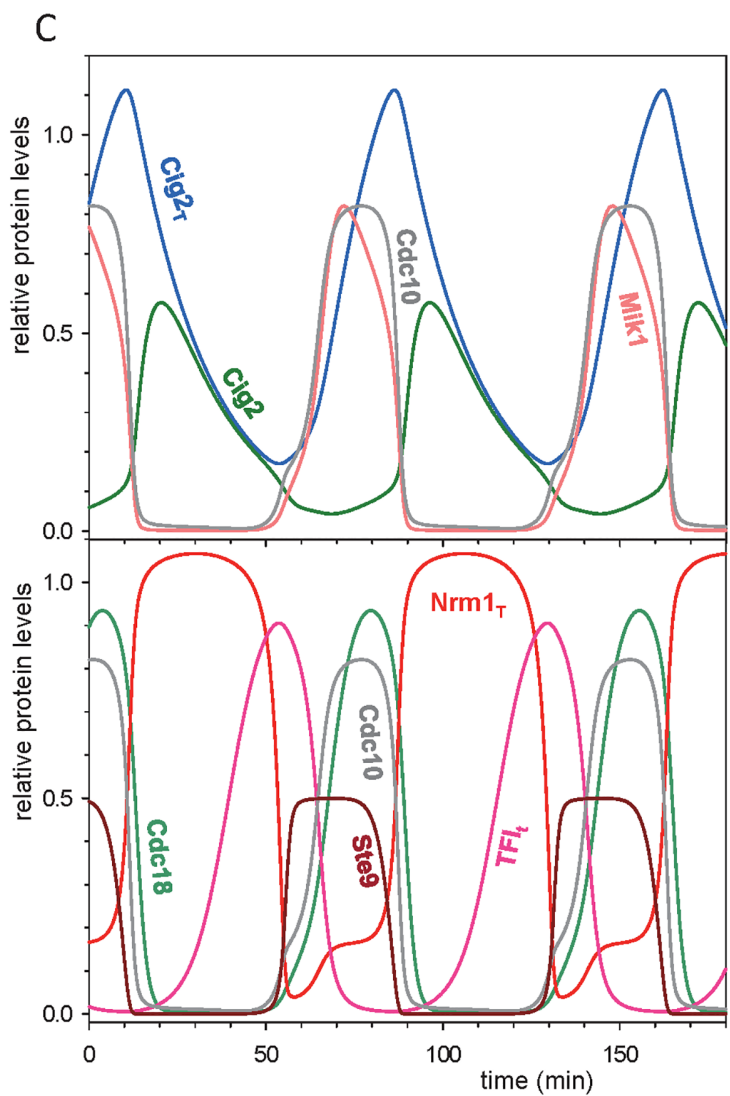

FIGURE 3: The transcriptional negative feedback regulated by two positive feedback loops. (A) Influence diagram.

(B) Phase plane portrait: both $\mathrm{Nrm}_{\mathrm{T}}$ and $\mathrm{Cig} 2_{\mathrm{T}}$ display bistability, as indicated by their $\mathrm{N}$ - and Z-shaped balance curve, respectively. The limit cycle oscillation is indicated by the dotted closed orbit. (C) Numerical simulation of the network shown in A.

oscillations might drive once-and-only-once DNA replication, we present a simple model that distinguishes four different forms of replication origins (Figure 4A). Origins are loaded with $\mathrm{MCM}$ helicase complexes by Cdc18 during the licensing step $(U \rightarrow L)$, while licensed origins are fired by Cdk1 activity. Firing origins $(F)$ initiate bidirectional replication, thereby leaving behind unlicensed, replicated origins (R), which are refractory for relicensing because of the Cdk1 activity associated with them (Wuarin et al., 2002). The Cdk1dependent block of relicensing $(R \rightarrow U)$ is competitively inhibited by Cdc18, but only when Cdc18 level is unphysiologically high. When coupled to the LFRU model of replication origins, our model of Cig2-kinase oscillations can drive discrete rounds of complete DNA duplications, as indicated by the temporal separation of origin licensing and firing rates (Figure 4B). During every oscillation of Cig2 activity, all replicated/refractory (R) origins are converted into licensed $(L)$ origins and vice versa (Figure $4 B$ ).

\section{Control by the DPR}

During endoreplication, Cig2-kinase oscillations drive discrete rounds of DNA synthesis with the periodicity of cell mass doubling time (Kiang et al., 2009), which suggests a strong coupling between the oscillatory mechanism in Figure 3 and the DPR. The DPR oscillates because it increases sharply during DNA replication and declines slowly by cell growth outside S phase. It is reasonable to assume that endoreplication (when cells skip mitosis) is controlled by the DPR at the G1/S transition when Cdc10 is activated, by relieving it from inhibition by TFI. In general, the period of the Cig2-kinase oscillation becomes adjusted to the cell mass doubling time if a component in the Cdc10 transcriptional network mirrors the DPR. One possibility is an "inhibitor-dilution mechanism" (Fantes et al., 1975), for which the DPR is mirrored by the falling concentration of $\mathrm{TFI}$. This mechanism requires that the TFI synthesis rate is dictated by gene-dosage rather than by the size of the cell (Schmoller et al., 2015; Heldt et al., 2018).

An inhibitor-dilution model is explored in Supplemental Figure S2. Supplemental Figure S2A illustrates how the total concentration of TFI affects the dynamic properties of the network. The network can only oscillate at low TFI concentrations. When $[\mathrm{TFI}]_{T}$ is higher than a threshold level (the green arrow in Supplemental Figure S2A), the stable solution of the network is a stable G1 steady-state (" $\mathrm{G} 1$ arrest"), with inactive Cdc10 transcription factor. The stable steady state is created near the origin of the phase plane (low Cig2 and Nrm1 levels) by the intersection of the two balance curves (Supplemental Figure S2B). In this model (constant TFI synthesis rate during growth), [TFI $]_{T}$ is steadily decreasing as the cell grows, and the cell's dynamic state is slowly drifting to the left on Supplemental Figure $\mathrm{S} 2 \mathrm{~A}$. Once $[\mathrm{TFI}]_{\mathrm{T}}$ drops below the threshold of the stable steadystate regime (the red arrow), the network starts to oscillate by activation of Cdc10. The rise of Cdk1:Cig2 activity initiates DNA replication, which results in duplication of the TFI gene and a doubling of its rate of synthesis. This will cause an abrupt increase of total TFI concentration (assuming fast TFI turnover), which pushes the dynamic state of the network back above the green arrow, where it becomes attracted to the stable G1 steady-state again. Numerical simulations show that this TFI-dilution mechanism only results in DPR-controlled endoreplication cycles if the TFI level 
A

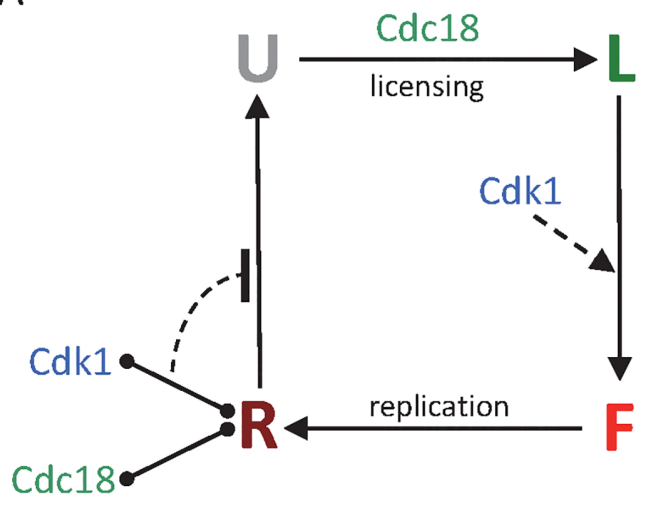

B

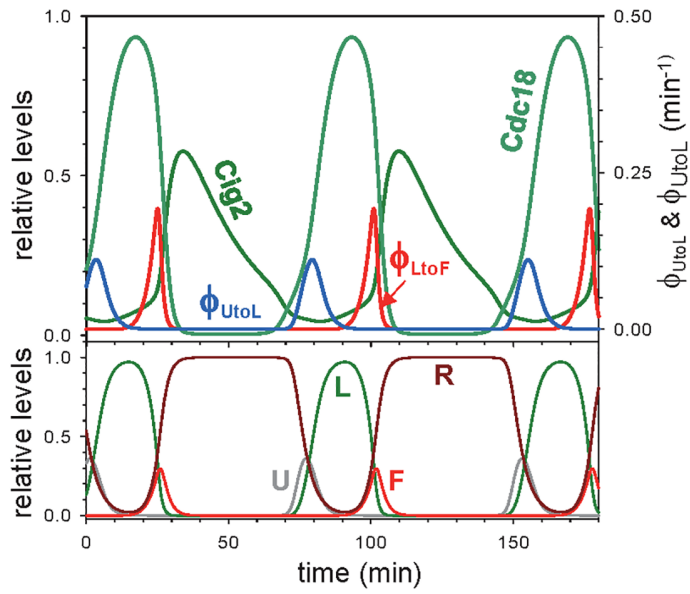

FIGURE 4: Dynamics of DNA replication driven by an oscillation in Cdc18 abundance and Cig2-kinase activity. (A) Reaction mechanism for interconversion of various forms of replication origins (U-unloaded, L-loaded, F-firing, and R-replicated/refractory) by $\mathrm{Cdc} 18$ and Cdk1-kinase (see text). The $\mathrm{L} \rightarrow \mathrm{F}$ and $\mathrm{R} \rightarrow \mathrm{U}$ steps are highly sensitive to $\mathrm{Cdk} 1$ activity. The dumbbells denote competitive binding of Cdk1 and Cdc18 to replicated origins. (B) Numerical simulation of the DNA replication mechanism on panel A driven by the Cig2-oscillatory mechanism on Figure 3. The fluxes $\phi$ through steps $\mathrm{U} \rightarrow \mathrm{L}$ (licensing) and $\mathrm{L} \rightarrow \mathrm{F}$ (firing) indicate a clear temporal separation of origin licensing and activation.

rapidly mirrors changes of DPR (as in Supplemental Figure S2C). However, rapid change of TFI concentration requires that the TFI protein is unstable, which does not seem to be the case for budding yeast Whi5, which has a half-life longer than $6 \mathrm{~h}$ (Schmoller et al., 2015).

We rather favor a "titration-mechanism" that postulates a starter kinase (SK) whose number of molecules per genome mirrors the inverse of DPR (Figure 5A). We envision a SK molecule that binds strongly to specific binding sites on chromosomes, where it phosphorylates and inactivates TFI in complex with Cdc10. SK molecules accumulate in an amount proportional to cytoplasmic content (e.g., ribosomes) and bind to a number of sites that is proportional to nuclear chromosome number. This mechanism makes the rate of TFI inactivation by SK proportional to the inverse of DPR, and it leads to Cdc10 activation above a critical inverse-DPR value. One of the kinases up-regulated by $\mathrm{Cdc} 10$ also phosphorylates and inactivates $\mathrm{TFI}$, and this positive feedback loop causes an abrupt entry of the cell into S phase. Figure 5B illustrates how the total concentration of SK affects the dynamic properties of the network. At low [SK], there exists only a stable steady state of low Cig2-kinase activity (G1 arrest). As the cell grows, [SK] eventually crosses a threshold (the red arrow in Figure 5B), past which the G1-arrested state disappears and the control system sets off on an oscillation. As before, rising Cig2kinase activity initiates DNA replication, which doubles the number of chromosomal binding sites and halves the number of SK molecules per genome, pushing the control system back into the domain of attraction of the stable G1 steady state. Numerical simulation of this titration-mechanism provides a robust DPR-controlled endoreplication cycle (Figure 5C), where the temporal period of DNA replication is identical to the mass doubling time of the cells.

\section{The DNA-replication checkpoint}

In the previous section, we showed how oscillations in the DPR can entrain the transcriptional oscillator to the mass doubling time of cell growth. High values of the DPR (i.e., small cell size relative to DNA content) stabilize a G1-arrested state, that is, a stable steady state of low activity of Cdc10 transcription factor and Cig2-kinase activity. In this section we discuss the effect of a "replication checkpoint" on the Cig2-kinase oscillation. Inhibition of DNA replication by hydroxyurea leads to stabilization of both total Cig2 level and the phosphorylated (inhibited) form of Cdk1:Cig2 (Zarzov et al., 2002; Hermand and Nurse, 2007), as well as dissociation of Nrm1 from MBF (de Bruin et al., 2008). Experiments also suggest that Cdc18 is necessary for maintenance of inhibitory phosphorylation of Cdk1 and thereby for the replication checkpoint (Hermand and Nurse, 2007). We implement these experimental findings in our model (see Figure 6A) by supposing that unreplicated DNA (1) inhibits Cig2kinase induced degradation of Mik1 in a Cdc18-dependent manner and (2) increases the equilibrium dissociation constant between $\mathrm{Cdc10}$ and $\mathrm{Nrm} 1$. These parameter changes influence both balance curves in the following ways (see Figure 6B). The [Cig2 $]_{T}$ balance curve (green) becomes horizontal, because Cdc10 inhibition by $\mathrm{Nrm} 1$ is compromised. The $\mathrm{N}$-shaped characteristic of the Nrm1 balance curve (red) is lost because of the weaker antagonism between Cig2-kinase and Mik1. These changes of the balance curves create a new intersection point with high Cig2 level (but low Cig2kinase activity) and intermediate Nrm 1 level, which defines a stable steady state corresponding to the replication checkpoint.

\section{Entraining Cig2-kinase activity to Cdc13-kinase oscillations}

Another important question concerns the mechanism that couples the Cdk1:Cig2 and Cdk1:Cdc13 oscillators during mitotic cycles. The strict alternation of Cdk activities driving S-phase and M-phase of the mitotic cycle (Figure 1A) requires suppression of the Cdk1:Cig2 oscillator by Cdk1:Cdc13 kinase during G2 and M phases. The overlapping substrate specificity of C $\mathrm{dk} 1$ :cyclin complexes provides a simple solution for tight coupling of the two oscillators. By making both Ste9 and TFI phosphorylable substrates of Cdk1:Cdc13, the Cdk1:Cig2 oscillator becomes sensitively dependent on the absence of the mitotic kinase (Figure 6C), consistent with fundamental observations by Hayles et al. (1994). With increasing Cdk1:Cdc13 activity, the large amplitude Cig2-oscillations are damped down to a stable steady state (Figure 6C). This steady state, created by Cdk1:Cdc13 phosphorylation of Ste9, is located in the bottom-right corner of the Nrm1-Cig2 phase plane (Figure 6D), with high Nrm1 and low Cig2 levels that are characteristic of G2 cells. The stable steady state is created by flattening the Nrm1 balance curve, a consequence of Cdc13-dependent inactivation of 
A

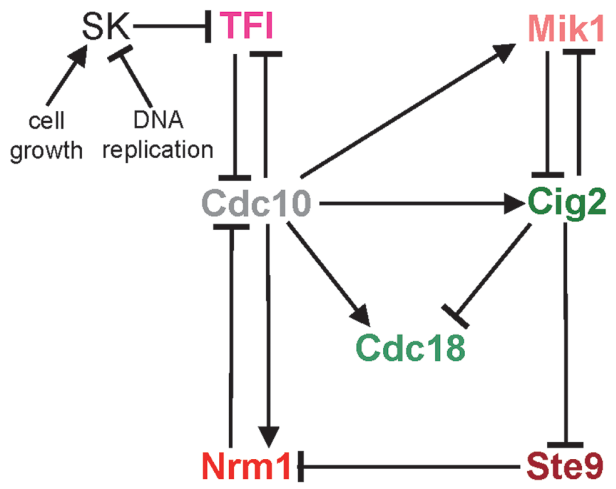

B

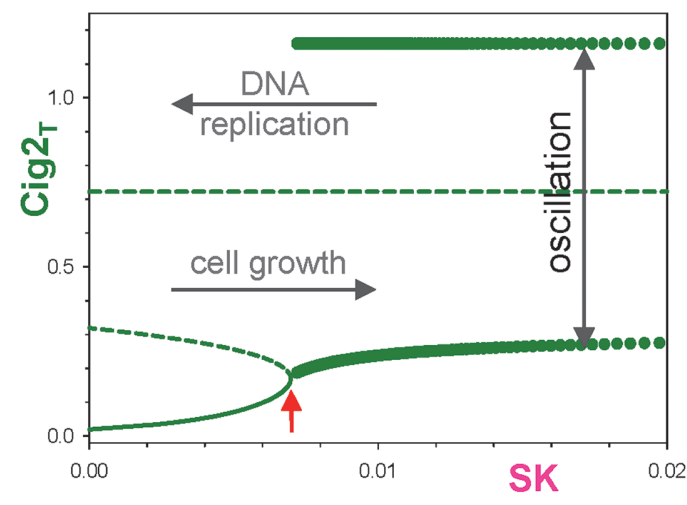

C

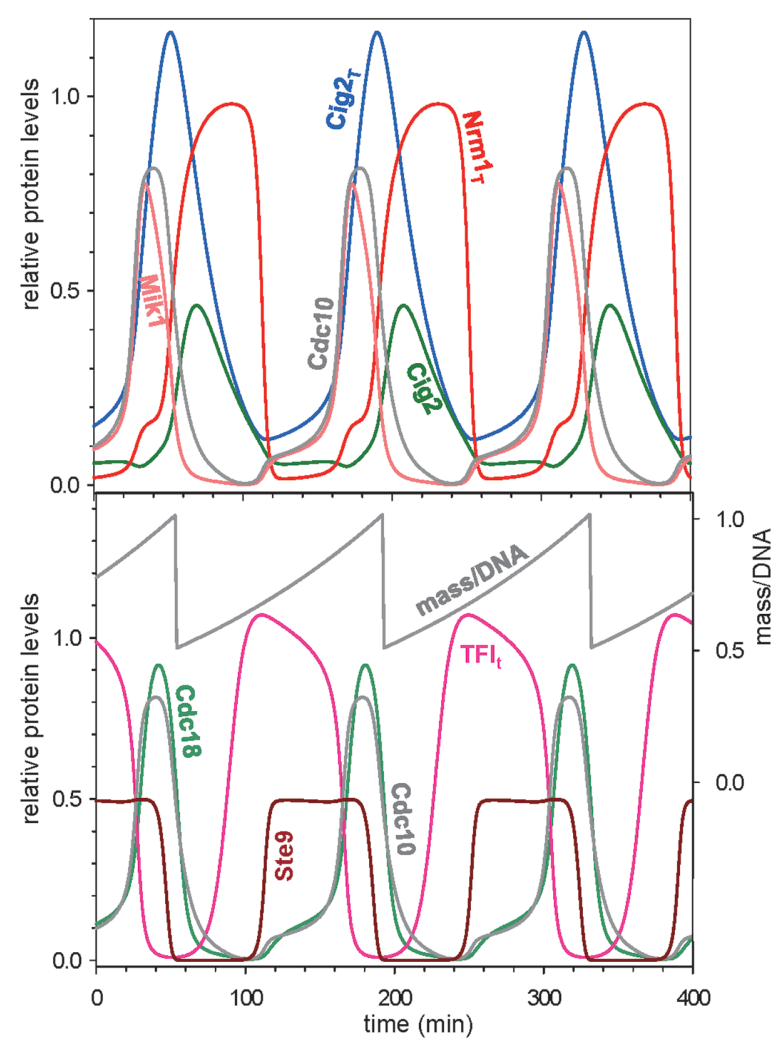

FIGURE 5: Oscillation controlled by DPR. (A) Influence diagram of the network with a SK whose activity is controlled by the DPR. Cell growth increases the number of SK molecules, and DNA replication doubles the number of SK-binding sites on the genome. (B) The effect of SK level on the regulatory network. Small SK levels create stable steady states (solid line), but high levels of SK allow large amplitude oscillations (the filled circles are minima and maxima). Unstable steady states are indicated by dashed lines. (C) Numerical simulation of the network.

Ste9, which stabilizes Nrm1. In the presence of Cdc13 (mitotic cyclin), the Cig2-oscillator cannot complete a full cycle, because the stable steady state in Figure 6D represents a G2-M "checkpoint." Only after mitotic degradation of Cdc13 will this checkpoint be removed, allowing the Cig2-oscillator to proceed into a G1-like state. This reasoning demonstrates that our model of endoreplication can also explain the strict alternation of $\mathrm{S}$ and $\mathrm{M}$ phases during mitotic cycles.

\section{Uncoupling of DNA replication from mitosis in other situations}

In addition to depletion of Cdc13 mitotic cyclin, multiple rounds of DNA replication in the absence of mitosis can be induced by overexpression of several cell cycle regulators (cdc18 op, rum1op, or ste9op) in $\mathrm{cdc}^{13^{+}}$genetic background (Moreno and Nurse, 1994; Nishitani and Nurse, 1995; Yamaguchi et al., 1997; Kitamura et al., 1998; Yanow et al., 2001). Rum1 is a strong stoichiometric inhibitor of Cdk1:Cdc13 complex (Correa-Bordes and Nurse, 1995); Ste9/ Srw1 is an activator of APC/C-dependent degradation of Cdc13 (Yamaguchi et al., 1997; Kitamura et al., 1998); Cdc18 plays a role in the inhibition of Cdk1:cyclin by the unreplicated DNA checkpoint (Hermand and Nurse, 2007). Therefore, overexpression of any one of these three cell cycle regulators has the ability to eliminate or countermand Cdc13-kinase activity and create a situation similar to Cdc13-depletion. However, overexpression of these cell cycle regulators has pleiotropic effects because of their influence on other proteins in the network (Figure 7A), and these effects must be taken into consideration in the model. While Ste9 has a direct pleiotropic effect in our network by promoting Nrm1 degradation, both Ste9 and Rum 1 have indirect effects by weak inhibition of Cdk1:Cig2 kinase (Correa-Bordes and Nurse, 1995; Yamaguchi et al., 1997; Benito et al., 1998). Furthermore, very high expression of Cdc18 causes strong inhibition of Cdk1:Cdc13 activity because excessive binding of Cdc18 to Cdk1 out-competes other Cdk1 substrates (Greenwood et al., 1998).

The effect of overexpression of these cell cycle regulators can be conveniently illustrated on a Signal-Response (S-R) diagram where the Signal is the extent of protein overexpression and the Response is one of the network components (we choose the total Cig2 level). The S-R diagrams for overexpression of Cdc18, Rum1 or Ste9, shown in Supplemental Figure S3, are similar because the effects of overexpression on the Nrm1-balance curve (the red curves in Figure $7 B, C$ and D) are similar, as we discuss below. Referring again to Supplemental Figure S3, we see that, at low expression levels of these proteins, the Cig2-oscillator is arrested in a G2/M-like state, as is the usual case during mitotic cycles, when Ste9 is inhibited by the mitotic Cdc13-kinase. This stable steady state, with low Cig2 level, is only observable during mitotic cycles, and its elimination is required for uncoupling DNA replication from mitosis. In the middle regime of the bifurcation diagrams in Supplemental Figure S3, the two chromosomal events ( $\mathrm{S}$ and $\mathrm{M}$ ) become uncoupled, and the Cig2-control network commences limit cycle oscillations similar to Cdc13-depleted cells. In all three situations, the oscillations of Cdk1 activity drive complete rounds of genome duplication with 

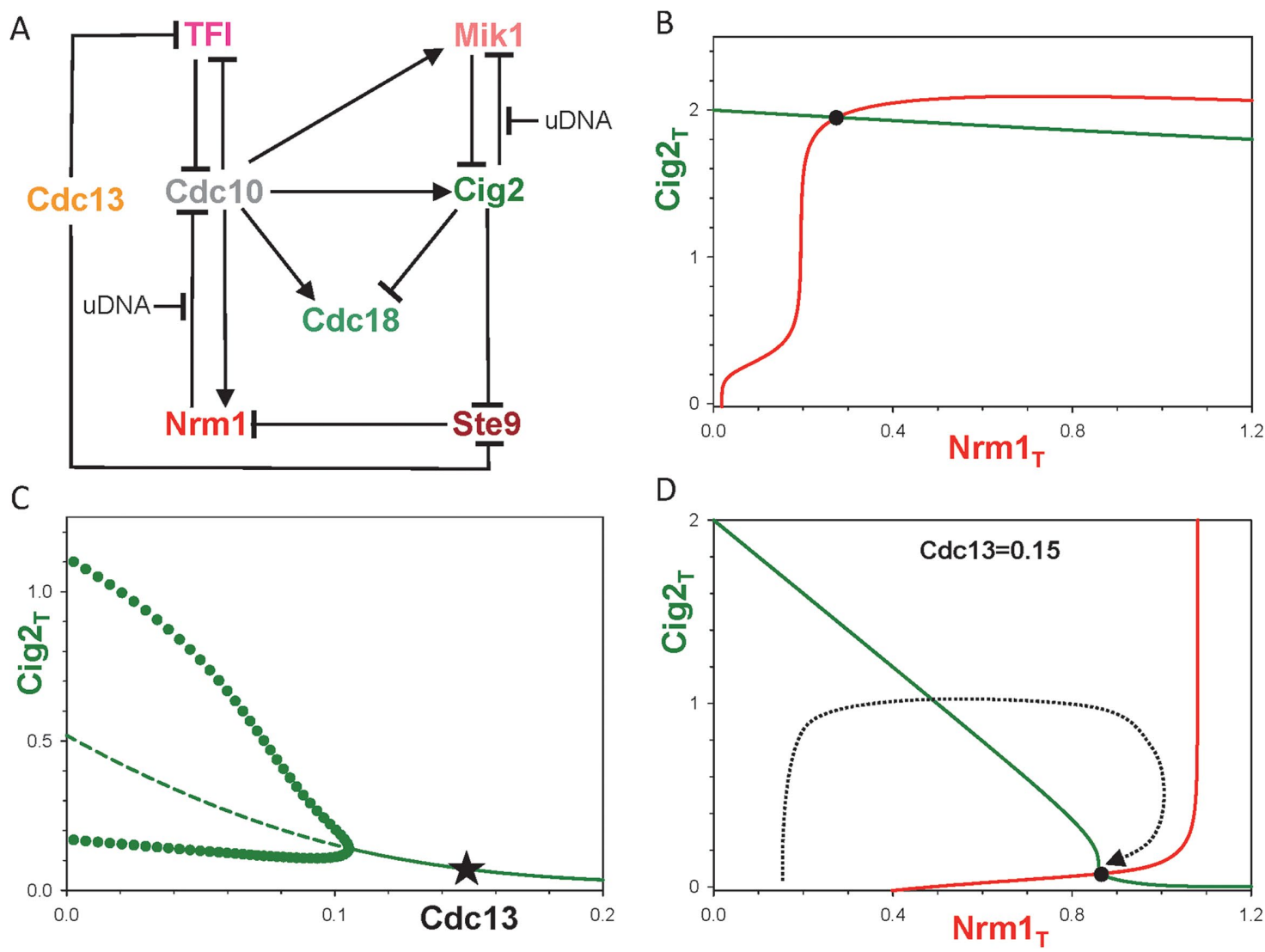

FIGURE 6: The effect of the replication checkpoint and of Cdc13 mitotic cyclin on the Cig2-kinase oscillation. (A) The effects of Cdc13-kinase and unreplicated DNA on the network. (B) Unreplicated DNA creates a stable steady state (๑) corresponding to the replication checkpoint. (C) Increasing activity of Cdc13-kinase causes a gradual decrease of the amplitude of Cig2 oscillations (indicated by filled circles), until oscillations terminate in a stable steady state (solid line) by a Hopf-bifurcation. (D) Higher than threshold level of Cdc13 (indicated by the star in C) creates a stable steady state corresponding to a G2-like state (Cig2 level low, Nrm1 high).

temporally separated origin licensing and firing, which is illustrated for medium (20-fold) Cdc18 overexpression in Figure 8A. In the oscillatory regime, the two balance curves of the phase plane (Figure $7 C$ and D) intersect on the middle branch of the N-shaped Nrm1balance curve in an unstable steady state. Despite similar phase plane portraits and S-R diagrams, the temporal dynamics of ste9op and rum $1^{\text {op }}$ oscillations are quite different (Supplemental Figure S4). The oscillations in ste9op are indistinguishable from the cdc13-depleted situation (Figure 5). On the other hand, overexpression of Rum1 significantly inhibits Cig2-kinase activity, thereby delaying DNA replication and the accumulation of Nrm1

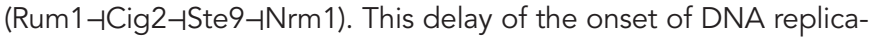
tion causes a significant ( $40 \%)$ increase of cell size-to-DNA ratio in rum1 ${ }^{\mathrm{OP}}$ cells compared with cdc13-depleted cells, in agreement with experimental data (Moreno et al., 1994; Kiang et al., 2009). According to our model, overproduction of Rum1 induces endoreplication with periodic appearance of the Cdc13 mitotic cyclin, which comes and goes because Ste9 periodically destroys it. This prediction could be tested by measuring Cdc13-GFP levels in single cells.

In the oscillatory regime, the unstable steady state, at the intersection of the two balance curves on Figure 7, shifts to higher Cig2 $\mathrm{T}$ and lower $\mathrm{Nrm}_{\mathrm{T}} \mathrm{in}$ proportion to the extent of overexpression of the modulated protein (Cdc18, Rum1, or Ste9). The shift of the unstable steady state is caused by a lifting of the N-shaped Nrm1balance curve (the red curve in Figure 7), because high levels of these regulators of Cdk1 activity make it harder for Cdk1:Cig2 to inactivate Ste9 than in Cdc13-depleted cells. Eventually, the rise of the Nrm1-balance curve destroys the limit cycle oscillations, above a critical level of overexpression, thereby creating a third regime where the control system settles in stable steady state of high level of $\mathrm{Cig}_{\mathrm{T}}$ (Supplemental Figure S3). Although the total level of Cig2 is high, the activity of Cdk1:Cig2 kinase is moderated by tyrosine phosphorylation of Cdk1 by Mik1. Cig2-kinase activity is below the threshold for Cdc10 inhibition, so the Cdc10-targeted genes (cdc18, cig2, and mik1) are highly expressed. Nonetheless, the Cig2-kinase activity must be high enough to support DNA replication in these mutant cells, as indicated by their phenotypes.

For example, the phase plane on Figure 7B illustrates the intersection of the Cig2 and Nrm1 balance curves for a high level of Cdc18 overexpression, sufficient to create a stable steady state with high Cig2 and low Nrm1 levels. We associate this stable steady state with the overreplication phenotype characteristic of cdc18 cells (Nishitani and Nurse, 1995; Yanow et al., 2001) with simultaneously licensed and activated replication origins (Kiang et al., 2010). In this steady state, the protein levels of Cdc18, Cig2, and Mik1 are all up-regulated, thereby creating a condition where both the loading factor (Cdc18) and the activator (Cig2-kinase) of DNA replication coexist permanently (Figure 8B). Consequently, the temporal separation of origin licensing and firing, which depends on Cig2-kinase oscillations, is lost, and origins are continually being licensed by Cdc18 and fired by Cig2-kinase in a disorganized manner in these cells. 

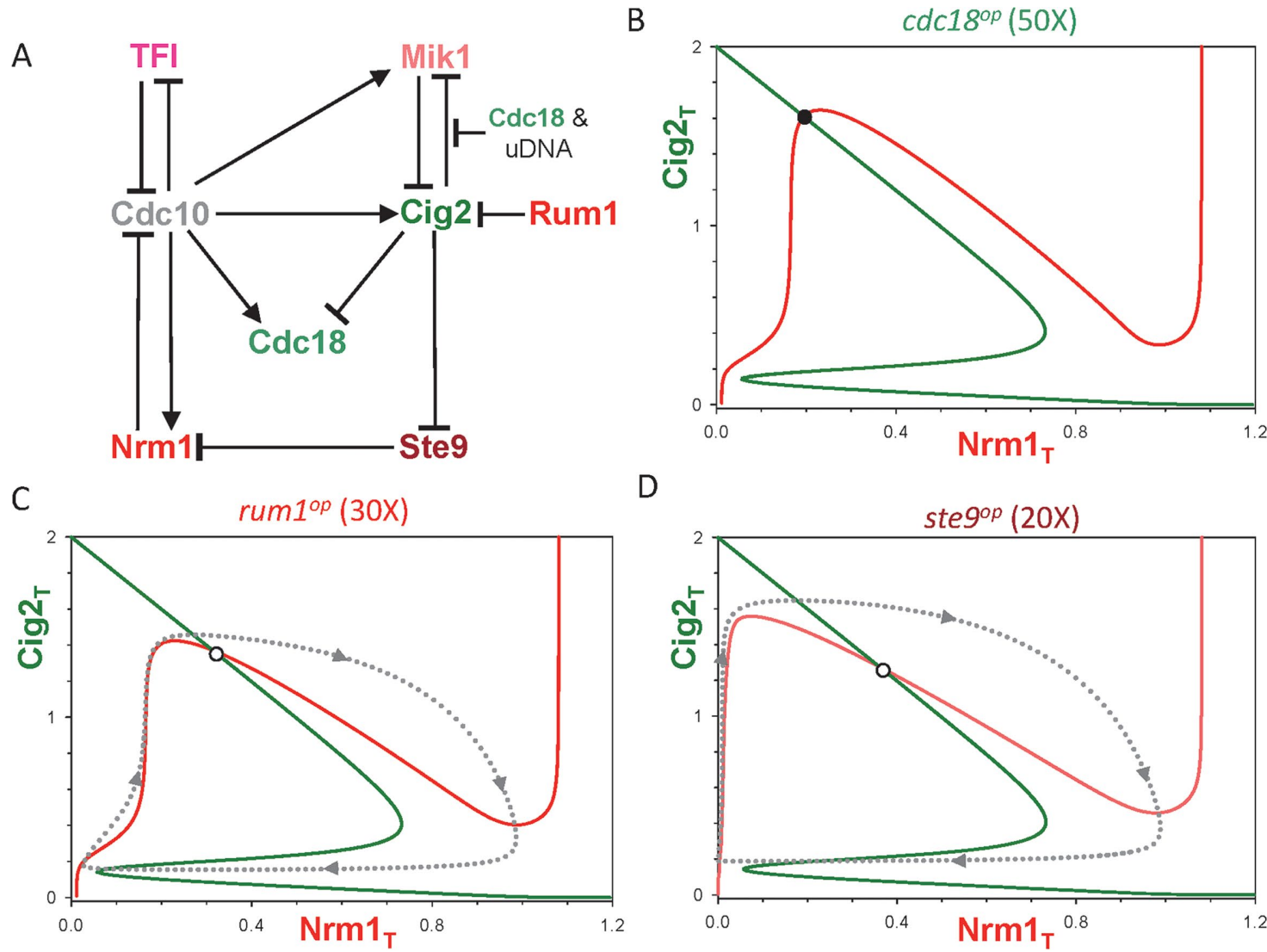

FIGURE 7: Re-replication in overexpressing strains. (A) Influence diagram. Phase plane portraits for overexpression of cdc18 (B), rum1 (C), and ste9 (D). In all cases, overexpression of proteins increases the N-shaped Nrm1T balance curve compared with a Cdc13-depleted strain (Figure 3B). The levels of overexpression of Rum1 and Ste9 in C and D drive oscillatory endoreplication cycles, corresponding to the oscillatory regimes of Supplemental Figure S4. The cdc18 ${ }^{\circ}$ level in $B$ locks the network in a stable steady state with high-Cig2 $T$ and low-Nrm1 levels, which we associate with DNA overreplication.
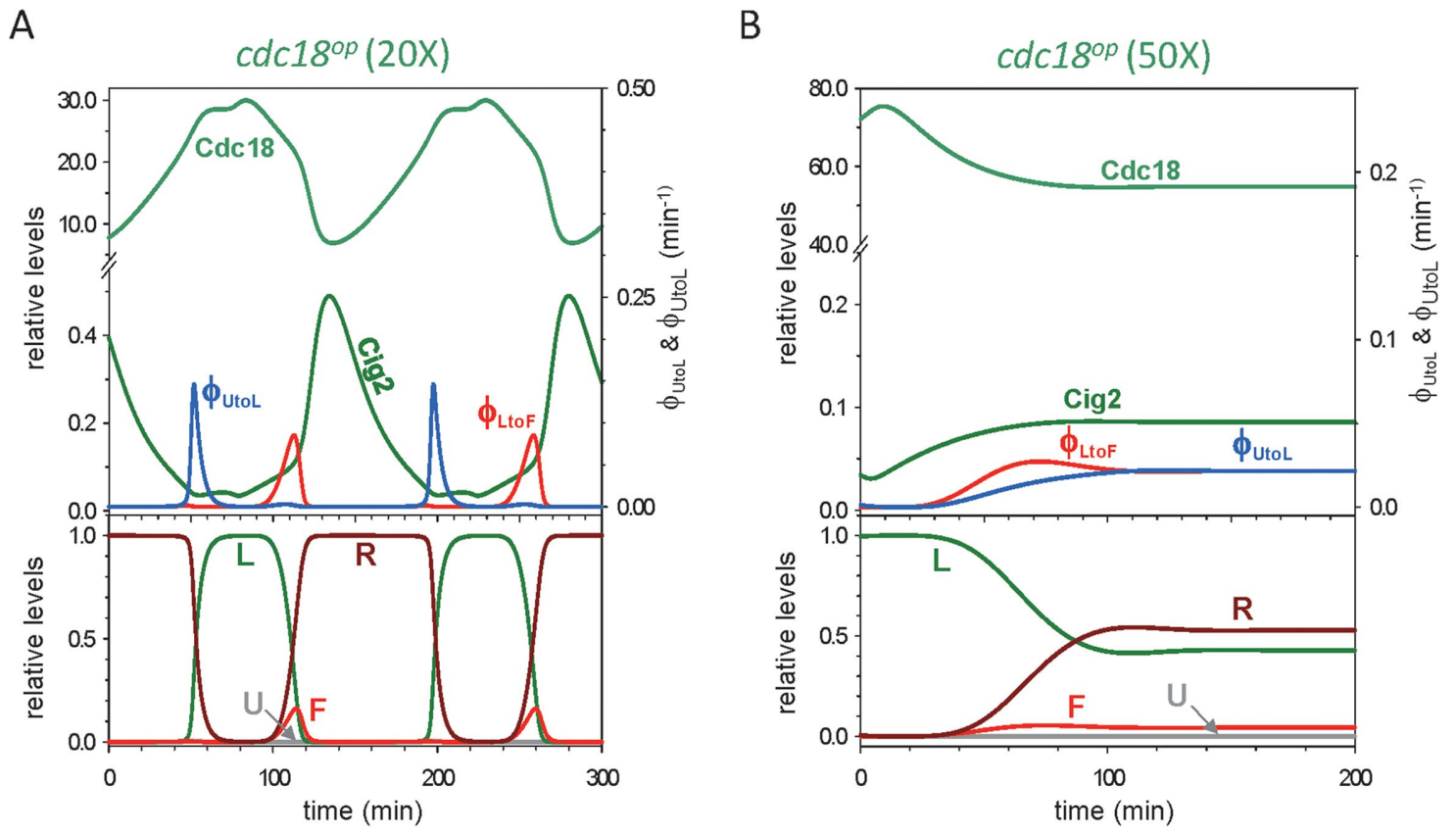

FIGURE 8: DNA replication in Cdc18 overexpressing cells. Numerical simulations of the DNA replication mechanism of Figure 4A for endoreplicating (A) and overreplicating (B) $c d c 18^{\circ p}$ cells. Licensing and firing fluxes are temporally separated at medium (20-fold) level of overexpression (A); they become steady and identical at high (50-fold) level of overexpression (B). 
To be more precise, we note that the steady state at 50 -fold overexpression of $\mathrm{cdc} 18^{+}$has $\mathrm{Cig} 2 \mathrm{~T} \approx 1.55, \mathrm{Cig} 2 \approx 0.088$ (because Cig2-kinase activity is depressed by Mik1 to 5\%), and Cdc18 $\approx 55$. These levels of Cdc18 and Cig2-kinase maintain a steady flux of $\sim 0.02 \mathrm{~min}^{-1}$ in origin licensing and firing (Figure 8B), which suggests a doubling of DNA content every $50 \mathrm{~min}$. Observe the similarities of the steady states of Cdc18-overexpressing cells (Figure 7B) and of S phase-arrested cells by hydroxyurea (Figure 6B); in both cases, $\mathrm{Cig}_{\mathrm{T}}$ is high and $\mathrm{Nrm}^{\mathrm{T}} \mathrm{T}$ is low. The difference is that hydroxyurea blocks DNA synthesis by depletion of its precursors, while, in cdc18 ${ }^{\circ}$ cells, replication is ongoing.

According to our model (see Supplemental Figure S3), endoreplication transitions to overreplication with increasing levels of protein overexpression in all three mutants cdc18 $\mathrm{p}$, rum10p, and ste9op. The only difference between overreplicating rum1op and ste9op cells compared with Cdc18 overexpression is that the steady-state flux of origin licensing and firing is $\sim 0.008 \mathrm{~min}^{-1}$ (DNA doubling every $\sim 120 \mathrm{~min}$ ). Overexpression of gene transcription in fission yeast is accomplished using the thiamine-repressible $n m t$ promoter (e.g., nmt-rum 1+); overexpression is induced by removing thiamine from the growth medium. Since it takes many hours for fission yeast cells to metabolize residual, intracellular thiamine, the level of overexpression of the mutant gene rises steadily over the induction period. It is likely, therefore, that, as the protein level increases, there is a continuous shift of growing cells from mitotic cycles, to DPR-regulated endoreplication cycles, to continuous overreplicative genome expansion. Our model suggests the possibility of endoreplication for $c d c 18^{\circ}$ cells during early induction and overreplication for rum $1^{\text {op }}$ and ste9op cells at very high expression levels. These phenotypes have not yet been observed; they are predictions of the model that could be investigated experimentally.

\section{DISCUSSION}

During normal growth and proliferation, fission yeast cells exhibit strict alternation of DNA replication and mitotic division in order to maintain their DPR within strict bounds, an approximately twofold range around an optimal ratio. The timing of DNA synthesis and mitosis during the fission yeast cell cycle is controlled by fluctuating activities of Cig2-dependent kinase and Cdc13-dependent kinase, respectively. Their oscillations are strongly coupled so that there is one peak of each kinase activity in each cell cycle; furthermore, the oscillations of DNA replication and cell division are strongly entrained to cell growth, so that wild-type fission yeast cells exhibit a stable and narrow distribution of cell length at division $(14 \pm 0.1 \mu \mathrm{m})$

These properties of fission yeast cell reproduction can be compromised in mutant strains. If mitotic cyclin (Cdc13) is sufficiently depleted, then nuclear and cell division are eliminated, but DNA replication continues by periodic genome doubling in concert with mass doubling (Kiang et al., 2009), a phenomenon called DPRcontrolled "endoreplication." During endoreplication, origins of replication are periodically licensed (bound by Cdc18 loading factor), fired (phosphorylated by Cig2-kinase activity), delicensed (by degradation of $\mathrm{Cdc} 18$ ), and then relicensed (by activation of Cdc10, a transcription factor for $\mathrm{Cdc18}$ and Cig2). This sequence of events is periodically initiated by oscillations of Cdc10 transcription factor, as the mutant cells undergo endoreplication cycles: $\mathrm{G} 1 \rightarrow \mathrm{S} \rightarrow \mathrm{G} 2 \rightarrow \mathrm{G} 1 \rightarrow \ldots$. Overexpression of $\mathrm{Cdc} 18$ in fission yeast induces overreplication of the genome without periodic execution of G1/S transitions (Nishitani and Nurse, 1995). In cells overexpressing Cdc18, DNA origins cannot be delicensed, and high lev- els of Cig2 protein in these strains cause continuous, disorganized replication of chromosomes.

In this work, we have presented a new dynamic model of endoreplication cycles in fission yeast cells. At the heart of our model, the Cdc10 transcription factor is regulated by two NFLs (see Figure 1B). Cdc10 up-regulates synthesis of its own stoichiometric inhibitor, Nrm1; this is a short (two-component) NFL. Additionally, Cdc10 drives synthesis of $\mathrm{Cig} 2$, and Cig2-kinase inactivates an E3 ubiquitin ligase (APC/C:Ste9) that labels Nrm1 for degradation by proteasomes (Figure 1B); this is a four-component NFL. Because neither of these NFLs can oscillate on its own, we supplement them with a positive feedback on Cig2-kinase activity by inhibitory phosphorylation of the kinase subunit ( $\mathrm{Cdk} 1$, see Figure 2A). This addition creates an oscillation with temporal separation between DNA replication licensing (by Cdc18) and initiation (by Cig2). An additional (hypothetical, but likely) positive feedback controls the activation of Cdc10 by a TFI (Supplemental Figure S1A). This addition provides a means to couple the oscillator with cytoplasmic growth (Figure 5A). These DPR-controlled oscillations drive multiple, discrete rounds of DNA replication (Figure 5) that we associate with endoreplication cycles observed in Cdc13-depleted fission yeast cells (Hayles et al., 1994).

In our earlier model for the endoreplication cycle (Novak and Tyson, 1997), Rum1, a G1 regulatory protein, played a central role in the oscillatory mechanism. Rum1 is a stoichiometric inhibitor of Cdk1:cyclin complexes, a stronger inhibitor of Cdk1:Cdc13 than of Cdk1:Cig2. Rum1 degradation is promoted by Cdk:cyclin-dependent phosphorylation (Benito et al., 1998). In the early model, Cig2 level was regulated by a NFL, while its activity was amplified by the double-negative (i.e., "positive") feedback between Rum1 and Cig2-kinase. In the present model, the role of Rum1 (to inhibit Cdk1:Cig2 kinase activity) is replaced Mik1 (and possibly by Wee1 as well), a protein kinase that phosphorylates Cdk1 and inhibits its kinase function. Rum1 is not part of the core Cig2 oscillatory mechanism driving endoreplication cycle (Figure $5 \mathrm{~A}$ ) in the present model. We propose that Rum1, although it regulates the activity of Cdk1:Cig2, is not necessary to drive Cig2 oscillations.

In addition to depletion of $\mathrm{Cdc13}$ mitotic cyclin, multiple rounds of DNA replication in the absence of mitosis can be induced by overexpression of several cell regulators ( $c d c 18^{\circ p}$, rum 10p or ste9op) in $c d c 13^{+}$genetic background. Interestingly, each of these proteins (Cdc18, Rum1 and Ste9), besides being a strong Cdk1:Cdc13 inhibitor, has significant pleiotropic effects on other components of the network, for example, inhibition of Cdk1:Cig2. As a result of such additional effects, our model suggests that mutants overexpressing these proteins do not necessarily correspond to the oscillatory behavior (periodic endoreplication) characteristic of Cdc13depleted cells; rather, sufficiently high levels of overexpression induce overreplication of the genome.

We explain the difference between endoreplication and overreplication in terms of our dynamical model using the concept of balance curves on a phase plane. Periodic endoreplication is understood to be a limit cycle oscillation on the Nrm1-Cig2 phase plane (Figure 3B) derived from an unstable steady state at the intersection of the Nrm1 (red) and Cig2 (green) balance curves. The limit cycle oscillations of Cig2 activity drive a temporal separation in the licensing and firing of DNA replication origins, which results in discrete rounds of exact duplication of genome content (Figure 4). DNA overreplication in cells overexpressing $\mathrm{Cdc} 18$ suggests that $c d c 18^{\circ} \mathrm{p}$ cells are stuck in an unusual state where the licensing and firing of DNA replication origins occur simultaneously and continuously (Figure 8B). In our model, constitutive overexpression of Cdc18 
raises the $\mathrm{N}$-shaped $\mathrm{Nrm} 1$-balance curve until a stable steady state is created in the upper left corner of the phase plane (Figure 7B). In this steady state, the protein levels of Cdc10-targeted genes (cdc18, mik1, and cig2) are all up-regulated. We associate this steady state (with high Cig2, high Cdc18, and low Nrm1 levels) to an overreplication phenotype. This observation leads us to conclude that endoreplication and overreplication are distinguished by a qualitative change of the dynamical system (a "bifurcation," in the language of dynamical systems theory) (Tyson and Novak, 2020).

\section{MATERIALS AND METHODS}

All mathematical equations and computational methods are described and explained in detail in the Supplemental Text. We also provide the code for each model in the form of ".ode" files (see the Supplemental ODE Files) that allow the users to reproduce our figures with the free available software XPP/AUTO (http:// www.math.pitt.edu/ bard/xpp/xpp.html).

\section{ACKNOWLEDGMENTS}

We are grateful to Jacky Hayles and Sergio Moreno for profitable discussions and to Adrien Wald for his contribution at an early stage of the project. We acknowledge financial support from BBSRC Strategic LoLa grant BB/M00354X/1 to B.N.

\section{REFERENCES}

Ayte J, Schweitzer C, Zarzov P, Nurse P, DeCaprio JA (2001). Feedback regulation of the MBF transcription factor by cyclin Cig2. Nat Cell Biol 3, 1043-1050.

Benito J, Martin-Castellanos C, Moreno S (1998). Regulation of the G1 phase of the cell cycle by periodic stabilization and degradation of the p25rum1 CDK inhibitor. EMBO J 17, 482-497.

Bertoli C, Skotheim JM, de Bruin RA (2013). Control of cell cycle transcription during G1 and S phases. Nat Rev Mol Cell Biol 14, 518-528.

Blanco MA, Sanchez-Diaz A, de Prada JM, Moreno S (2000). APC(ste9/srw1) promotes degradation of mitotic cyclins in $\mathrm{G}(1)$ and is inhibited by cdc2 phosphorylation. EMBO J 19, 3945-3955.

Christensen PU, Bentley NJ, Martinho RG, Nielsen O, Carr AM (2000). Mik1 levels accumulate in $\mathrm{S}$ phase and may mediate an intrinsic link between S phase and mitosis. Proc Natl Acad Sci USA 97, 2579-2584

Correa-Bordes J, Nurse P (1995). p25rum1 orders S phase and mitosis by acting as an inhibitor of the p34cdc2 mitotic kinase. Cell 83, 1001-1009.

Coudreuse D, Nurse P (2010). Driving the cell cycle with a minimal CDK control network. Nature 468, 1074-1079.

de Bruin RA, Kalashnikova TI, Aslanian A, Wohlschlegel J, Chahwan C, Yates JR, 3rd, Russell P, Wittenberg C (2008). DNA replication checkpoint promotes G1-S transcription by inactivating the MBF repressor Nrm1. Proc Natl Acad Sci USA 105, 11230-11235.

Diffley JF (2004). Regulation of early events in chromosome replication. Curr Biol 14, R778-R786.

Diffley JF (2011). Quality control in the initiation of eukaryotic DNA replication. Philos Trans R Soc Lond B Biol Sci 366, 3545-3553.

Fantes PA, Grant WD, Pritchard RH, Sudbery PE, Wheals AE (1975). The regulation of cell size and the control of mitosis. J Theor Biol 50 , 213-244.

Fisher DL, Nurse P (1996). A single fission yeast mitotic cyclin B p34cdc2 kinase promotes both S-phase and mitosis in the absence of $\mathrm{G} 1$ cyclins. EMBO J 15, 850-860.

Gerard C, Tyson JJ, Coudreuse D, Novak B (2015). Cell cycle control by a minimal Cdk network. PLoS Comput Biol 11, e1004056.

Greenwood E, Nishitani H, Nurse P (1998). Cdc18p can block mitosis by two independent mechanisms. J Cell Sci 111 (Pt 20), 3101-3108.

Hayles J, Fisher D, Woollard A, Nurse P (1994). Temporal order of S phase and mitosis in fission yeast is determined by the state of the p34cdc2mitotic B cyclin complex. Cell 78, 813-822.

Heldt FS, Lunstone R, Tyson JJ, Novak B (2018). Dilution and titration of cell-cycle regulators may control cell size in budding yeast. PLoS Comput Biol 14, e1006548.
Hermand D, Nurse P (2007). Cdc18 enforces long-term maintenance of the $S$ phase checkpoint by anchoring the Rad3-Rad26 complex to chromatin. Mol Cell 26, 553-563.

Jallepalli PV, Brown GW, Muzi-Falconi M, Tien D, Kelly TJ (1997). Regulation of the replication initiator protein $\mathrm{p} 65 \mathrm{cdc} 18$ by CDK phosphorylation. Genes Dev 11, 2767-2779.

Kelly TJ, Martin GS, Forsburg SL, Stephen RJ, Russo A, Nurse P (1993). The fission yeast cdc18+ gene product couples $S$ phase to START and mitosis. Cell 74, 371-382.

Kiang L, Heichinger C, Watt S, Bahler J, Nurse P (2009). Cyclin-dependent kinase inhibits reinitiation of a normal S-phase program during $\mathrm{G} 2$ in fission yeast. Mol Cell Biol 29, 4025-4032.

Kiang L, Heichinger C, Watt S, Bahler J, Nurse P (2010). Specific replication origins promote DNA amplification in fission yeast. J Cell Sci 123, 3047-3051.

Kitamura K, Maekawa H, Shimoda C (1998). Fission yeast Ste9, a homolog of Hct1/Cdh1 and Fizzy-related, is a novel negative regulator of cell cycle progression during G1-phase. Mol Biol Cell 9, 1065-1080.

Kominami K, Toda T (1997). Fission yeast WD-repeat protein pop1 regulates genome ploidy through ubiquitin-proteasome-mediated degradation of the CDK inhibitor Rum1 and the S-phase initiator Cdc18. Genes Dev 11, 1548-1560.

Lundgren K, Walworth N, Booher R, Dembski M, Kirschner M, Beach D. (1991). mik1 and wee1 cooperate in the inhibitory tyrosine phosphorylation of cdc2. Cell 64, 1111-1122.

Martin-Castellanos C, Blanco MA, de Prada JM, Moreno S (2000). The puc1 cyclin regulates the $\mathrm{G} 1$ phase of the fission yeast cell cycle in response to cell size. Mol Biol Cell 11, 543-554.

Millar JB, Lenaers G, Russell P (1992). Pyp3 PTPase acts as a mitotic inducer in fission yeast. EMBO J 11, 4933-4941.

Mondesert O, McGowan CH, Russell P (1996). Cig2, a B-type cyclin promotes the onset of $\mathrm{S}$ in Schizosaccharomyces pombe. Mol Cell Biol $16,1527-1533$

Moreno S, Hayles J, Nurse P (1989). Regulation of p34cdc2 protein kinase during mitosis. Cell 58, 361-372.

Moreno S, Labib K, Correa J, Nurse P (1994). Regulation of the cell cycle timing of Start in fission yeast by the rum $1+$ gene. J Cell Sci Suppl 18, $63-68$.

Moreno S, Nurse P (1994). Regulation of progression through the $G 1$ phase of the cell cycle by the rum1+ gene. Nature 367, 236-242.

Morgan DO (2007). The Cell Cycle: Principles of Control, New Science Press: London.

$\mathrm{Ng}$ SS, Anderson M, White S, Mclnerny CJ (2001). mik1(+) G1-S transcription regulates mitotic entry in fission yeast. FEBS Lett 503, 131-134.

Nishitani H, Lygerou Z, Nishimoto T, Nurse P (2000). The Cdt1 protein is required to license DNA for replication in fission yeast. Nature 404, 625-628.

Nishitani $H$, Nurse P (1995). p65cdc18 plays a major role controlling the initiation of DNA replication in fission yeast. Cell 83, 397-405.

Novak B, Tyson JJ (1997). Modeling the control of DNA replication in fission yeast. Proc Natl Acad Sci USA 94, 9147-9152.

Novak B, Tyson JJ (2008). Design principles of biochemical oscillators. Nat Rev Mol Cell Biol 9, 981-991.

Nurse P (1975). Genetic control of cell size at cell division in yeast. Nature $256,547-551$.

Nurse P (1990). Universal control mechanism regulating onset of M-phase. Nature 344, 503-508.

Reymond A, Marks J, Simanis V (1993). The activity of S.pombe DSC-1-like factor is cell cycle regulated and dependent on the activity of p34cdc2. EMBO J 12, 4325-4334.

Russell P, Nurse P (1986). cdc25+ functions as an inducer in the mitotic control of fission yeast. Cell 45, 145-153.

Russell P, Nurse P (1987). Negative regulation of mitosis by wee1+, a gene encoding a protein kinase homolog. Cell 49, 559-567.

Rustici G, Mata J, Kivinen K, Lio P, Penkett CJ, Burns G, Hayles J, Brazma A, Nurse P, Bahler J (2004). Periodic gene expression program of the fission yeast cell cycle. Nat Genet 36, 809-817.

Schmoller KM, Turner JJ, Koivomagi M, Skotheim JM (2015). Dilution of the cell cycle inhibitor Whi5 controls budding-yeast cell size. Nature 526, 268-272.

Stern B, Nurse P (1996). A quantitative model for the cdc2 control of $S$ phase and mitosis in fission yeast. Trends Genet 12, 345-350. 
Tyson JJ, Csikasz-Nagy A, Novak B (2002). The dynamics of cell cycle regulation. Bioessays 24, 1095-1109.

Tyson JJ, Novak B (2020). A Dynamical Paradigm for Molecular Cell Biology. Trends Cell Biol 30, 504-515.

Wood E, Nurse P (2015). Sizing up to divide: mitotic cell-size control in fission yeast. Annu Rev Cell Dev Biol 31, 11-29.

Wuarin J, Buck V, Nurse P, Millar JB (2002). Stable association of mitotic cyclin B/Cdc2 to replication origins prevents endoreduplication. Cell $111,419-431$.

Yamaguchi S, Murakami H, Okayama H (1997). A WD repeat protein controls the cell cycle and differentiation by negatively regulating Cdc2/B-type cyclin complexes. Mol Biol Cell 8, 24752486.

Yamaguchi S, Okayama H, Nurse P (2000). Fission yeast Fizzy-related protein srw1p is a $\mathrm{G}(1)$-specific promoter of mitotic cyclin $\mathrm{B}$ degradation. EMBO J 19, 3968-3977.

Yanow SK, Lygerou Z, Nurse P (2001). Expression of Cdc18/Cdc6 and Cdt1 during $\mathrm{G} 2$ phase induces initiation of DNA replication. EMBO J 20, 4648-4656.

Zarzov P, Decottignies A, Baldacci G, Nurse P (2002). G(1)/S CDK is inhibited to restrain mitotic onset when DNA replication is blocked in fission yeast. EMBO J 21, 3370-3376. 